\title{
Protée
}

\section{Le cinéma et l'empreinte du temps. L'oeuvre filmique au crible de la réception spectatorielle}

\section{Emmanuel Ethis}

Volume 27, numéro 2, 1999

La réception

URI : https://id.erudit.org/iderudit/030561ar

DOI : https://doi.org/10.7202/030561ar

Aller au sommaire du numéro

Éditeur(s)

Département des arts et lettres - Université du Québec à Chicoutimi

ISSN

0300-3523 (imprimé)

1708-2307 (numérique)

Découvrir la revue

Citer cet article

Ethis, E. (1999). Le cinéma et l'empreinte du temps. L'oeuvre filmique au crible de la réception spectatorielle. Protée, 27(2), 67-76.

https://doi.org/10.7202/030561ar
Résumé de l'article

Matière bien souvent impensée en ses dimensions anthropologiques les plus agissantes, le temps - fondation cachée de nos « boussoles culturelles »constitue une substance vive de l'oeuvre cinématographique. Observé sous cet angle, chaque récit filmique se laisse ainsi saisir comme le résultat d'une splendide synthèse temporelle qui n'a de cesse de se recomposer dans le regard du spectateur. De fait, l'introduction à la sociologie de la réception des oeuvres filmiques tente de rendre manifeste la diversité des appropriations du temps filmique qui replace les spectateurs face à des oeuvres réelles ; l'on voit, peu à peu, apparaître quelques-uns des traits saillants sur lesquels s'engage notre rapport à la cinématographie. 


\title{
LE CIN ÉM A ET L'EM PREINTE DU TEM PS L'đEUVRE FILMIQUE AU CRIBLE DE LA RÉCEPTION SPECTATO RIELLE
}

EMMANUEL ETHIS

\begin{abstract}
Jadis, la certitude d'obtenir à chaque instant de ma vie une révélation qui ne se renouvellerait plus composait le plus clair de mes secrets plaisirs: maintenant, je meurs honteux comme un privilégié qui aurait assisté à une fête sublime qu'on ne donnera qu'une fois. Chers objets, vous n'avez plus pour témoin qu'un aveugle qui meurt...
\end{abstract}

(Marguerite Yourcenar, Nouvelles Orientales)

Dans ses Entretiens sur le cinématographe ${ }^{1}$, Jean Cocteau assimile le cinéma à une sorte de muse assez suspecte, «[...] en ce sens qu'il lui est impossible d'attendre alors que toutes les autres muses attendent et devraient être peintes ou sculptées dans l'attitude de l'attente». Il ajoute

[...] les gens se plaignent toujours de lenteurs ou de longueurs dans une œuvre à laquelle ils assistent pour la première fois. Outre que cela vient souvent de leur faiblesse d'attention aux lignes profondes, ils oublient que les classiques fourmillent de longueurs et de lenteurs qu'ils admettent parce que ce sont des classiques.

Le langage du poète n'est certes pas celui du sociologue ou du philosophe de l'esthétique qui, l'un et l'autre, pourraient assurément user du terme d' «horizons d'attente» pour qualifier cette observation conséquente d'un effet de classicisation. En l'occurrence, selon Gérard Genette la relation à une œuvre d'art suppose

[...] la reconnaissance de l'intention esthétique, ou «candidature à l'appréciation» qui la définit comme œuvre, et la prise en compte de sa position relative dans un champ historique et générique qui module son action: je ne reçois pas de la même façon une dissonance chez Mozart et chez Webern,

un tableau selon que j'y vois un authentique Vermeer ou un habile pastiche, ni une description selon qu'elle me vient d'un roman ou d'un récit documentaire. Cette relativité culturelle s'ajoute à la réalité subjective de l'appréciation qu'elle accentue plutôt qu'elle ne l'annule. ${ }^{2}$

De fait, en déplaçant la problématique de l'esthétique à distance des objectivismes spontanés, Genette tente de rendre cette relation à «sa liberté, à son risque et à 
son plaisir». Mais que signifie exactement le sens de cette restitution si elle ne se joue pas en deçà du jugement subjectif et temporaire du "c'est beau, c'est laid", c'est-à-dire si elle n'est pas «infra-appréciative»? Ne faut-il pas voir dès lors dans le problème des lenteurs et des longueurs dont parle Cocteau le témoignage d'une dimension plus objective et universelle de la relation qui va de l'œuvre au public et du public à l'œuvre? Parce que ce que nous valorisons dans l'appréciation courante du temps, singulièrement lorsqu'elle s'applique à l'œuvre filmique, et avant même sa hiérarchisation dans notre propre panthéon cinématographique, c'est une «intensité de mise en relation", une conjugaison plus ou moins achevée des temporalités d'un film et de son spectateur. L'acte de communication artistique est ainsi fait. La matière temporelle dont il se nourrit est décisive dans la passation du pacte qui liera ou non l'œuvre et son public et ce quelles que soient les nuances que l'on peut apporter à ce lien.

Expression symbolique d'un symbole ou constitution d'un cadre symbolique, le temps participe à un dispositif précaire où se déroulent dans leur vaste diversité les réceptions artistiques. Pour reprendre un mot cher à Roland Barthes, il joue sur le terrain de la "signifiance» et intervient à tous les stades de la relation à l'œuvre qui est à la fois

[...] communication d'un message et réinterprétation du message reçu, [et] dépend des codes et des compétences mis en jeu par la communication, des circonstances de la réception, des "attentes» des récepteurs et des "pactes de réception artistiques» qui se nouent en fonction de toutes ces données entre les destinataires de l'œuvre et les «marques» reconnues [...] pertinentes $d u$ message. ${ }^{3}$

Mais peut-on parler effectivement de pertinences temporelles d'une ouvre? En quoi constituent-elles une force formatrice et communicative de la relation au spectateur? Cette force est-elle assez "puissante» pour déposer une empreinte sur le spectre sociétal qu'un appareillage d'enquête serait susceptible de relever?

\section{LA SYMBOLIQUE TEMPORELLE AU CINÉMA, CLEF DES RÉCEPTIONS FILMIQUES?}

Si la conscience n'était pas la moindre partie de l'activité psychique, si elle était au courant de ses propres processus et de ses motivations, alors, quand elle "se fait croire», on pourrait l'accuser de mauvaise foi sartrienne. Si la croyance était un jugement rendu par la liberté cartésienne, les idéologies seraient des mensonges que nous nous ferions à nous-mêmes; seulement sentir n'est pas vouloir: il n'est pas en notre pouvoir que le miel nous paraisse doux ou amer, un théorème, vrai ou faux, et le roi, bon ou pas. Si la vérité était l'indice d'elle-même, l'impression qu'une chose fait sur notre esprit serait proportionnelle à la réalité de cette chose; en fait une croyance fausse, une erreur, peut avoir les mêmes effets qu'une vraie, et une vraie peut n'en avoir pas davantage. 4

Le temps pensé faussement comme une dimension objective est de cet ordre-là, et sa compréhension en tant que dimension ou cadre symbolique n'est ni indispensable, ni préalable à l'usage que l'on en fait. Car dans la réalité, tout se passe comme si le rapport d'inégalité, qui figure ci-dessous sur le schéma du temps articulé, était, en fait, traité en tous points comme un rapport d'égalité:

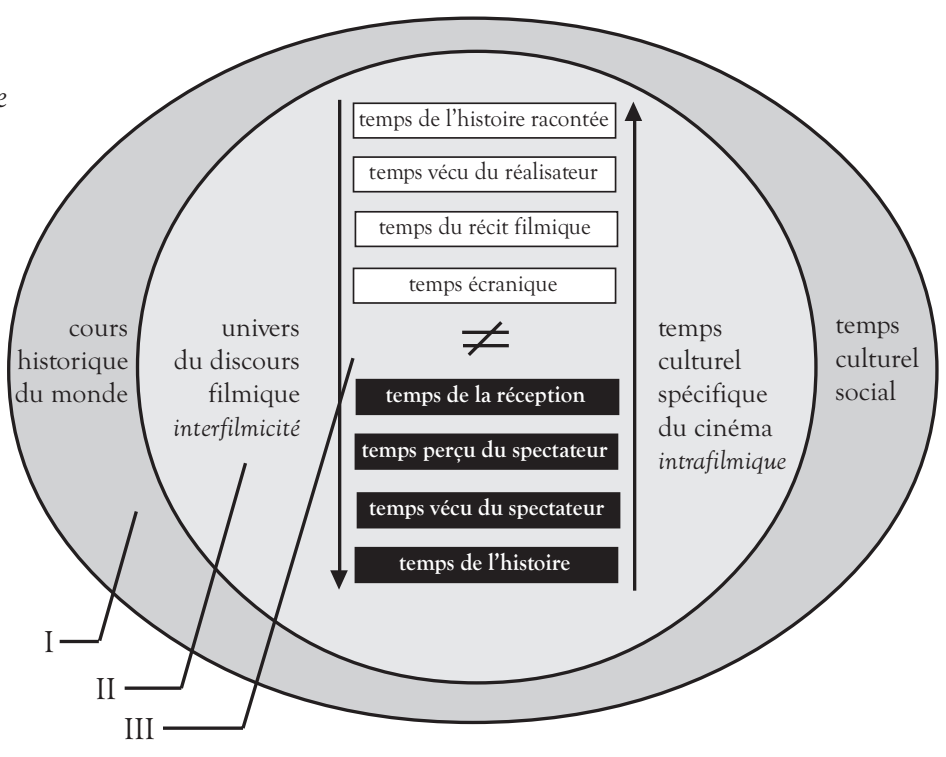


De l'histoire racontée à l'histoire telle qu'elle se reconstitue dans l'esprit du spectateur, l'épreuve du temps se joue sur au moins trois épaisseurs qui, si elles ont chacune une dynamique propre, restent éminemment interdépendantes les unes des autres. Première épaisseur (I): celle du cours historique du monde où se synthétise historiquement, socialement, et donc culturellement notre temps vécu et pensé. Deuxième épaisseur (II): celle de l'univers du discours filmique (ou si l'on veut du discours - qu'il soit pure invention ou documentaire réaliste - que le cinéma tient sur le monde) qui s'est, elle aussi, historiquement rationalisée au fur et à mesure que s'édifiait le patrimoine cinématographique. C'est sur la fréquentation de cet univers que l'on construit notre carrière de spectateur, une carrière qui nous est propre et dont on façonne les empreintes d'un film à l'autre (interfilmicité). Le temps vécu dans le cours historique du monde n'est pas le même que celui de l'univers du discours filmique. Il s'instruit sur des configurations conventionnelles qui en définissent la spécificité: c'est le temps intrafilmique. Enfin, dernière épaisseur représentée - entre les flèches ascendantes et descendantes de notre schéma -, celle qui s'institue à chaque rencontre cinématographique entre film et spectateur (III): un acte de communication complexe où se croisent les «horizons d'attente» des publics avec les qualités intrinsèques des œuvres qu'ils fréquentent. Se signent dans cet acte les conduites esthétiques d'où se dégagent cette «sorte de supplément invisible, cette intensification merveilleuse des rapports établis avec leur objet " ${ }^{5}$. Et la véritable question qui, par conséquent, se pose à toute analyse qui tenterait d'appréhender le lien entre les deux faces ${ }^{6}$ du fait cinématographique - le film et sa réception - trouve ici son origine.

Contrairement à une visite de musée où le visiteur auto-administre son temps, l'acte cinématographique impose la contrainte de sa temporalité au spectateur. On imagine qu'a priori les spectateurs doivent pouvoir évaluer assez justement la durée globale d'un film. Or, même lorsque l'objet qui leur est proposé est rituellement temporalisé comme c'est le cas des séries télévisées qui sont toutes calibrées sur un format de 52 minutes, on observe que plus de $60 \%$ des individus à qui l'on demandait "combien de temps dure un épisode de la série "The X-files», nous répondaient un temps qui n'était résolument pas le bon (cf. tableau ci-après).

Variations dans l'évaluation de la durée d'un épisode de
The X-files
\begin{tabular}{|c|c|c|c|c|c|}
\hline temps & $\begin{array}{c}\text { inférieur de } \\
\text { plus de } 20^{\circ}\end{array}$ & $\begin{array}{c}\text { inférieur } \\
\text { de } 10\end{array}$ & égal & $\begin{array}{c}\text { supérieur de } \\
20 \text { ' et plus }\end{array}$ & total \\
\hline $\begin{array}{c}\text { Effectif } \\
\text { en \% }\end{array}$ & 17 & 28 & 38 & 17 & 100 \\
\hline
\end{tabular}

Bien plus qu'un constat troublant, on saisit dans cet exemple singulier la difficulté d'évaluer ses propres pratiques, même celles qui semblent les plus délimitées par une durée systématique. Mieux, 34\% des individus ajoutent ou retranchent plus de 20 minutes à The X-files, soit une durée qui excède pratiquement la moitié du temps effectif de l'épisode. Les enquêtés, toutes générations confondues, connaissaient pourtant très bien cette série dont toutes les saisons, depuis cinq ans, ne cessent d'être rediffusées et popularisées en France.

Que peut-on apprendre de cette déroute dans les perceptions temporelles? En premier lieu, qu'elle suffit à sanctionner le rapport d'inégalité entre temps écranique et réception spectatorielle et des termes qui leur sont antécédents dans l'épaisseur III du schéma de la page ci-contre: l'expérience de la temporalité incorporée dans la narration filmique traduit la nature instable du message «filmique» lui-même. Même dans ses processus narratologiques les plus rationalisés, le travail cinématographique s'élabore toujours sur une série d'allers-retours entre les épaisseurs I, II et III, où cours historique du monde et univers du discours filmique sont des étendues où le temps culturel est hétérogène; une hétérogénéité qui se transpose clandestinement dans les interprétations du monde et de ce qu'on en dit manipule, positivement, un temps dissous et invisibilisé.

La détermination du temps repose sur la capacité humaine de mettre en relation deux ou plusieurs séquences différentes de transformations - nous dit Norbert Élias -, l'une servant d'échelle de mesure du 
temps pour l'autre ou pour les autres. Ce type de synthèse représente une performance qui est loin d'être élémentaire, puisque la séquence de référence peut être profondément différente de celle à laquelle elle sert d'échelle de mesure. 7

Quelle est donc la séquence de référence que l'on utilise intuitivement lorsque l'on se confronte à un objet aussi temporalisé que le film de cinéma ou de télévision? Qu'est-ce qui nous fait évaluer sa durée de manière tellement variable? Qu'est-ce qui en substance nous fait dire à la sortie du cinéma qu'un film était long ou court?

Jean-Claude Passeron qualifie de réception faible le pacte que nous entretenons avec les images ${ }^{8}$. Cette qualification s'appuie selon ce dernier sur le caractère non assertorique de toute icône, généralement oublié dans la plupart des actes d'interprétation ou d'analyse d'une image: une image est incapable d'asserter comme le fait une phrase. Et, le fait filmique en est tout aussi incapable ${ }^{9}$, et ce même quand la condition sine qua non à la passation d'un pacte interprétatif de la part du spectateur implique que ce dernier se mette dans la posture de celui à qui l'on va raconter une histoire. Au demeurant, ceux à qui on raconte une histoire filmique, même très courte, ne la voient ni ne la comprennent de la même manière. C'est une particularité du récit oral ou écrit que d'inciter son auditeur ou lecteur à y rapporter ou y projeter des éléments de sa propre vie afin de combler les vides laissés par la narration, de donner une substance vitale aux mots pour que s'ancre pour chacun le sens de l'histoire; c'est à cette particularité que l'on attache parfois la variation d'interprétation du sens narratif. Mais l'analyse de ce sens-là ne s'épuise pas non plus dans le récit filmique qui pourtant semble saturer la substance narrative en y apportant des matériaux tels que le son ou l'image; ceux-ci ne réduisent en rien la multiplicité interprétative de l'œuvre. L'intention narrative que contient l'histoire filmée s'exprime dans une communication véritablement problématique.

La conscience intime du temps, dont parlait le philosophe Edmund Husserl ${ }^{10}$, trouve dans cette relation entre variation perceptuelle de la durée et narration filmique une résonance sémio-sociologique saillante. Bien entendu, il n'est pas question de réduire la réception des œuvres filmiques à l'unique réception temporelle. Si nos spectateurs sont des spectateurs du temps, ils ne regardent naturellement pas une matière temporelle "en-soi»: c'est la répercussion du temps de la narration filmique dans ce que «fabriquent" les publics qui nous intéresse ici. Elle permet d'armer un sérieux indice d'où l'on peut initier une enquête de réception. Elle donne aussi - ce n'est pas la moindre de ses qualités - un contour utile aux perceptions spectatorielles, car, contre toute attente, le temps nous livre, par son «caractère indépassable", quelques limites vraisemblables à l'interprétation filmique.

\section{LES FONCTIONS DE SOCIALISATION DES CEUVRES, PIERRE DE TOUCHE DE LA RÉCEPTION}

«La sociologie de la réception essaie de tirer toutes les conséquences du fait - souvent rappelé mais plus rarement exploré - que les œuvres picturales, musicales" ou cinématographiques

[...] comme les oeurres littéraires n'existent et ne durent que par l'activité interprétative de leurs publics successifs. S'attachant à

décrire les pactes de réception artistique qui caractérisent époques et publics, une sociologie, centrée sur le sort réservé à des œuvres singulières, se distingue donc de la sociologie de la consommation culturelle qui occupe aujourd'hui la plus grande place dans les enquêtes en sociologie de la culture. Elle s'intéresse en effet aux actes sémiques de l'expérience esthétique, picturale, musicale

ou cinématographique, «qu'elle vise à cerner par des indicateurs objectifs - ce en quoi elle relève pleinement de la sociologie d'enquête» ${ }^{11}$. D'emblée, en tentant de décrire le sens de la métamorphose du social depuis les configurations interprétatives de ceux qui vivent la culture, la sociologie de la réception complexifie terriblement les règles qui s'étaient surtout établies autour du repérage des dispositifs de reproduction d'attitudes et de classes, mécaniquement indexés aux résultats des enquêtes de consommation culturelle. Ainsi, à propos des œuvres filmiques, on pourrait, plutôt que de fonder une compréhension 
sociologique des comportements de spectateurs sur le box-office de leurs fréquentations cinématographiques, s'attacher à mettre l'accent sur les dimensions symboliques incorporées à l'œuvre - en l'occurrence le temps - et sur l'analyse des conduites sociales suscitées par celles-ci en ce qu'elles comportent toujours une dimension de connaissance et d'interprétation; en effet, l'espoir séminal de la sociologie de la réception est bien de réduire l'espace entre les approches institutionnelles de fréquentation et les études des processus stricto sensu de création artistique. Au demeurant, la sociologie de la réception se doit, comme toute sociologie de l'art digne de cette appellation, de satisfaire à ce «double contrat que son nom lui impose» 12 : ainsi,

[...] tout en offrant un rapport d'intelligibilité de même nature que pour d'autres domaines de la vie sociale, la connaissance sociologique de l'art doit s'engager à traiter des œeuvres en tant qu'ouvres et de leurs effets en tant qu'effets esthétiques. 13

Cette approche n'épuise évidemment pas tout ce qu'on serait en droit d'attendre d'un programme sociologique aspirant à traiter de façon exhaustive de la question filmique. Cependant, un tel programme est-il décemment envisageable? La question dominante et centrale à laquelle s'astreint la sociologie de la réception est celle de la passerelle qui va des productions filmiques à leurs recouvrements en symboles collectifs à travers des actes de consolidation et d'interprétation sociales. Par la force des choses, elle laisse donc en retrait deux autres grands types d'analyse développés en sciences sociales:

- les analyses de contenu des œuvres filmiques mises en relation avec l'espace social où elles se structurent et cela, indépendamment de leurs conditions de réception ${ }^{14}$;

- une sociologie du cinéma qui aurait pour but de décrire l'œuvre filmique au regard de ses consécrations et ses évolutions à la charnière d'un champ culturel et de la transformation d'un marché ${ }^{15}$.

Ainsi conçu, le travail sociologique qui tente d'investir l'œuure filmique en train de se faire s'inscrit dans le développement «naturel» des théories de la réception, telles qu'elles furent initiées par les Écoles de Prague et Constance; en ce sens, la thèse soutenue dans l'ouvrage fondateur de Hans Robert Jauss, Pour une esthétique de la réception ${ }^{16}$, soutient que la littérature et la plupart des formes artistiques modernes possèdent des histoires qui leur sont propres. Et pour cause, elles participent à part entière à la structuration de la réalité, structuration qui, ellemême, possède une dynamique et des effets singuliers. On ne peut donc «simplement» les assimiler à des arts de l'imitation comme c'était le cas dans les théories d'obédience marxistes et se contenter de les appréhender comme le décalque immédiat de l'expérience courante ${ }^{17}$. En déplaçant leur intérêt vers le procès de la lecture des œuvres, les théories de la réception débouchent sur un compromis positif entre l'histoire de l'art et la philosophie herméneutique, tout en reprenant pour la faire fructifier l'idée husserlienne d'une conscience dans la lecture des ouvres. Cette conscience est réifiée dans ce que Wolfgang Iser désigne par L'Acte de lire ${ }^{18}$, où l'œuvre est considérée comme une structure potentielle que le spectateur rend concrète en la mettant en rapport avec les normes et les valeurs sociales et culturelles qui l'environnent et par l'entremise desquelles il donne un sens à son expérience esthétique. En substance, lorsque nous sommes spectateurs d'une ouvre, notre attente est donc instruite en fonction de ce que nous avons déjà vécu en tant que spectateurs; néanmoins, l'impact d'événements inattendus est toujours possible, et ce sont généralement ceux-ci qui nous contraignent à reformuler tout ou partie de nos attentes et à faire évoluer notre vécu spectatoriel. Le rapport aux œuvres correspond ainsi à une stratégie de sauts en avant et en arrière d'où l'on tente de construire une configuration cohérente du sens général de notre expérience garanti par ces révisions perpétuelles. Et, comme l'indique Jacques Leenhardt [...] l'enjeu de ces déplacements est une vision dédoublée $d u$ processus de connaissance sociale. Connaître, en société, ce n'est pas seulement produire un savoir sur le monde, c'est aussi d'un même geste produire une image $d u$ monde. Penser la 
connaissance sociale comme une dialectique instable entre image $d u$ monde et savoir $d u$ monde, c'est s'interroger aussi bien rétroactivement sur ce que nous appelons «science sociale», que sur la fonction cognitive de l'imagination, comme activité sociale. 19

C'est donc en des termes de décalages produits avec la réalité, qu'il s'agira pour la sociologie de l'art de comprendre les significations de l'interprétation culturelle des œuvres, en l'occurrence des œuvres filmiques. En définitive, toute ouvre prise sous cet aspect communicationnel apparaîtra dans un double rapport dialectique avec la chaîne des œuvres qui la précèdent ou la suivent [interfilmicité] et avec celle des publics qui la reçoivent en lui donnant un sens. Et si le temps s'impose comme une limite de l'interprétation du message filmique, les théories de la réception viennent consolider doublement cette acception en le fédérant aux «représentations qui renforcent ou infléchissent le sens de ce qu'il véhicule».

\section{LA THÉORIE ESTHÉTIQUE DE LA RÉCEPTION}

Progressivement, l'activité interprétative du spectateur-destinataire quitte l'ombre dans laquelle la sémiotique structurale l'avait délaissée sous prétexte d'impureté méthodologique; elle n'est plus assimilée à cette «fiction scolastique» dont parlait Jakobson ${ }^{20}$, fiction qui la cantonnait à un acte strict de déchiffrement d'un code indépendant et détaché d'une communication authentique. Or, il faut désormais admettre que l'interconnexion de l'expérience vécue et de l'expression comme production de manifestations symboliques est la voie d'accès à la compréhension. De fait, les théories de la réception privilégient une ligne de convergence entre l'œuvre et le récepteur sur laquelle se concrétise l'expérience esthétique. Il faut cependant noter que cette "concrétisation" n'a pas forcément pour finalité de rendre expressément visible ce qui est au fondement de l'œuvre, comme le soutenait l'interprétation kantienne; les effets que l'œuvre produit sont à la croisée de deux plans constitutifs que Jauss désigne sous le concept d'horizons d'attente ${ }^{21}$ : l'horizon d'attente généré par l'œuvre elle-même et l'horizon d'attente social de ses publics. Le premier dessine, par le biais d'«un jeu d'annonces, de signaux - manifestes ou latents -, de références implicites, ou de caractéristiques déjà familières " 22 la prédisposition à un certain mode de réception. Le second correspond à la précompréhension du monde et de la vie qui est investie par le récepteur dans son activité; il y inclut les attentes concrètes qui s'accordent à l'horizon de ses intérêts, désirs, besoins, expériences tels qu'ils sont déterminés par la société, par sa carrière de spectateur, aussi bien que par son histoire individuelle qui scintille dans toute expérience. Il est, au reste, important de souligner que chaque horizon active sa propre temporalité: l'horizon d'attente de l'œuvre l'inscrit dans la dimension socioculturelle historique de sa création et l'horizon d'attente social l'actualise par l'interprétation renouvelée de chacun de ses spectateurs.

Le projet général de la réception consiste donc à comprendre en quoi réside la "fusion» 23 de ces horizons à la jonction desquels vont se réorganiser les différentes perceptions artistiques. Dans cette perspective, l'École de Constance s'est, pour sa part, principalement orientée vers une exploration historique des réceptions littéraires; en introduisant une sorte d'inventaire des différences, elle brave en partie l'idée du "plaisir pur qui doit être éprouvé par tout homme», en encourageant la rupture avec les dispositions socioculturelles singulières de la connaissance sensible jusqu'alors présupposées dans tout rapport aux œuvres: une ouvre d'art ne manifeste pas une impression exclusive, absolue et transcendante qui conditionnerait une contemplation indifférente à tout contexte socioculturel environnant.

Toutefois, si prometteuse soit-elle, l'esthétique de la réception de Jauss et des autres chercheurs de l'École de Constance n'est pas transposable telle quelle en tant qu'armature d'une enquête sociologique. De surcroît, si elle en esquisse la bonne direction, c'est précisément dans l'espace de la réception qu'elle laisse en friche que campe 
véritablement le questionnement sociologique. En effet, lorsque Jauss explique par exemple que le sens d'une ouvre doit être appréhendé dans le rapport que le spectateur tissera entre les normes et les valeurs externes à l'œuvre et l'œuvre elle-même, il ne précise ni le degré de liberté qu'a le spectateur de remplir les structures filmiques à partir de ses propres normes, ni depuis quelles instances symboliques de contrôle le film se laisse pénétrer. De plus, en rompant avec l'idée philologique du présent intemporel et éternel des œuvres, l'esthétique de la réception a déconstruit le concept d'interprétation exclusive. Néanmoins, si l'on s'accorde bien sur le fait qu'aucune œuvre n'a jamais été réalisée en vue de n'être décodée que par des spécialistes, les indicateurs qui nous permettraient d'accéder à l'expérience et aux conduites esthétiques du spectateur ne sont - sauf sur quelques grands pans du changement historique des horizons d'attente que faiblement investis. C'est dans son œuvre principale Pour une herméneutique littéraire ${ }^{24}$ que Jauss livre la construction appliquée la plus aboutie du déploiement théorique de son esthétique de la réception: son projet est une réhabilitation des droits de la jouissance $d u$ texte qui avaient été lésés jusque dans les théories modernes de l'art et notamment dans la Théorie esthétique d'Adorno qui l'a grandement inspiré. Adorno définit en effet l'art dans sa période moderne sous l'angle d'un rapport négatif à la société: c'est dans cette négativité que l'art conquiert à la fois son autonomie et sa fonction sociale profonde

[...] en se cristallisant comme chose spécifique en soi, au lieu de s'adapter aux normes sociales existantes et se qualifier comme "socialement utile», il critique la société par le simple fait qu'il existe. ${ }^{25}$

$\mathrm{Si}$ Jauss relève bien dans sa construction dialogique avec Adorno cette fonction d'émancipation de l'art moderne qui correspond effectivement à un changement historique d'horizon d'attente, il ne peut en revanche admettre cette imbrication trop forte avec l'idéologie pour laquelle l'art s'improvise signifiant et puise sa valeur dans son militantisme. Indirectement, ce qu'Adorno semble proposer dans cette thèse n'est autre, selon Jauss, qu'une variation contemporaine de l'esthétique platonicienne du beau: si le beau nous ravit, c'est que l'artiste a su par la mimesis se rapprocher progressivement d'un idéal qui, via la beauté du corps, touche la beauté de l'âme, qui, à son tour, doit nous mettre en relation avec la beauté première de l'Idée ${ }^{26}$. L'art renvoie toujours à autre chose que lui-même. En soi, il serait une idée imitée de second ordre. En réfutant cette thèse coercitive et restrictive de l'art, Jauss s'efforce de découvrir et de rétablir un espace élargi dans lequel peuvent coexister des formes plurielles et évolutives d'hédonismes esthétiques qui n'ont pas nécessairement besoin de passer par la reconnaissance légitimante de justifications idéelles ou culturelles périphériques. La jouissance esthétique qu'il revendique est infraesthétique: elle demeure un moyen sincère et profond de s'approprier le monde et de s'assurer soi-même.

La libération par l'expérience esthétique peut s'accomplir sur

trois plans: la conscience en tant qu'activité productrice crée un monde qui est son cuure propre; la conscience en tant qu'activité réceptrice saisit la possibilité de renouveler sa perception du monde; enfin - et ici l'expérience subjective débouche sur l'expérience intersubjective - la réflexion esthétique adhère à un jugement requis par l'oeurre, ou s'identifie à des normes d'action qu'elle ébauche et dont il appartient à ses destinataires de poursuiure la définition. 27

C'est du reste dans la poursuite de cette définition que s'autonomisent réellement les formes esthétiques, qu'elles se transforment et instaurent de nouveaux rapports de lecteurs aux œuvres en recréant de concert à chaque génération de nouvelles normes et de nouvelles valeurs ${ }^{28}$.

\section{POUR UNE RÉCEPTION SOCIOLOGISÉE DES CEUVRES}

Quand bien même l'on restaure, à l'instar de Jauss, l'intégrité des droits de la jouissance des ouvres, rien n'est plus compliqué que de rationaliser, ne serait-ce que de façon parcellaire, le jugement de goût porteur d'une appréciation esthétique. De fait, ce territoire d'ordinaire interdit sous prétexte qu'il est affaire d'émotions, voire de pures émotions, ne peut, hors 
des grandes bannières historiques agitées "d'authenticités légitimatrices", se laisser naturellement recueillir. En soi, l'expérience esthétique est aussi troublante que celle de la beauté vivante qui nous aimante irrésistiblement vers ces humains dont l'érotisme flamboyant et inexplicable nous dépasse et qui dépose en nous, pour reprendre les mots de Winckelmann, un sentiment fascinant d'humanité accomplie. Ces épreuves quotidiennes dont on dit couramment qu'elles sont de l'ordre de l'ineffable, mais dont on ne peut paradoxalement s'empêcher de le dire, ne peuvent que susciter une curiosité sociologique: comment un tel statut s'instruit-il? N'y a-t-il pas derrière l'estime accordée à une ouvre, lorsqu'elle est verbalement formalisée, un amalgame quelque peu énigmatique entre ce que Wittgenstein ${ }^{29}$ nomme un motif et une cause?

Être amené à réagir face à une œuvre d'art, c'est au mieux, et pour peu qu'on soit capable de le faire, argumenter sa propre conduite devant ce qu'elle contient de réalité expressive. En rebondissant sur un jugement de goût, cet argumentaire se trouve soudain dans l'impasse du mobile ou du motif qui prolongent verbalement cette expressivité.

Au tribunal, on vous demande quel est le motif de votre conduite et vous êtes supposé le connaître. À moins de mensonge, vous êtes supposé capable de dire quel est le motif de votre conduite. [...] On dit parfois: "personne ne peut voir en vous, mais vous, vous le pouvez", comme si étant si proche de vous-même, étant vousmême, vous connaissiez votre propre mécanisme. 30

Ainsi la sociologie de la réception opère-t-elle suivant une posture qui s'astreint à ne prêter à une œuvre rien qui n'émane effectivement de l'expression des actes sémiques $d u$ spectateur. Car c'est bien dans les comportements associés à une pratique réelle que la sociologie de la réception se propose de redessiner les contours des déterminations sociales qui peuvent, dans le cadre d'une analyse comparée, rendre véritablement justice du prix accordé aux œuvres. Et la découverte de ces déterminations ne peut en aucun cas faire l'économie d'une enquête qui soit - contrairement aux questionnaires d'audience ou aux inventaires de consommation culturelle spécifiquement calibrée aux configurations de l'expérience de la réception artistique. Les réceptions filmiques, dont il est ici question, vont donc reposer sur trois principes qui nous permettront de garantir quelques grandes lignes de comparabilité entre nos observations, les principes de spécificité, de singularité, de perceptibilité: $\left.1^{\circ}\right) \mathrm{Au}$ fondement du premier principe, la spécificité sémiologique du message filmique: elle tient à la combinaison spécifique de plusieurs matières de l'expression. Ceci contraint à analyser la logique d'une situation de réception qui n'est pas réductible sémiotiquement à la prise en compte des commentaires appréciatifs parlés qui ne maintiennent qu'une continuité expressive très distante avec le message audiovisuel tel qu'il est interprété en réalité:

[...] loin de relever de l'ordre du jugement, l'appréciation esthétique apparaît, lorsqu'on la restitue à sa genèse psychologique et sociale ou aux conditions de sa mise en acte, comme un acte sémique qui relève de l'ordre de l'interjection; elle tire son sens de la mimique, de la force apprise du «sentiment des règles", d'une "impression diffuse de correction", d'un mélange d'affect et de connaissances. Les actes de l'appréciation esthétique sont des additions de sens, "différents et différemment différents", même lorsqu'ils ont un air de famille, qui supposent pour signifier et persuader de leur signification, les circonstances où ils sont employés. 31

$2^{\circ}$ ) En observant les comportements de réception esthétique des œuvres filmiques par l'entremise d'un protocole d'enquête, il s'agit de respecter ensuite, uniment, le principe de singularité: ce principe impose d'effectuer une analyse à travers la réception d'œuvres singulières comme nous l'avons fait par exemple plus haut avec la série The X-files; «la réception artistique est, en effet, par définition perception et interprétation d'un message identifié dans l'individualité insubstituable de ses signifiants" ${ }^{32}$.

$3^{\circ}$ ) Enfin, partant de l'analyse platonicienne de l'hétérogénéité interne du plaisir, J.-C. Passeron compose un modèle descriptif qu'il étend au plaisir artistique:

[...] interpréter l'expérience qui se déclare et se vit en tant qu'expérience artistique comme le résultat d'un mélange de 
composantes hétérogènes qui doit précisément à sa mixité l'impression subjective de richesse insaisissable. 33

De cette hypothèse découle le principe de perceptibilité34 qui admet de n'attribuer à une ouvre que les aspects de cette ceuvre qui ont été perçus par des publics réels.

Une fois ces principes énoncés, du film à ses publics, une équation dotée d'une inconnue majeure semble peu à peu se dessiner. Cette inconnue qui court de l'œuvre filmique au spectateur et du spectateur à l'œuvre, c'est le fil du temps. Un fil du temps qu'il nous faut sociologiquement découdre si l'on veut observer comment il s'ourle sous les pliures qui conforment l'objet filmique aux lectures qui en sont faites. Car «l'équation temporelle se définit comme une configuration de traits communs aux individus qui participent d'une même culture " 35 . Une étude détaillée sur les comportements de gestion des temporalités quotidiennes, menée en fonction des catégories socioprofessionnelles ${ }^{36}$, a récemment montré que les ouvriers investissaient beaucoup moins bien leur temps de loisirs que les paysans: s'ils détiennent une connaissance accrue des temps étroitement mesurés, ils s'orientent temporellement plus mal que les hommes de la campagne. Pour sa part, Henri Mendras déclarait à propos des paysans:

Les unités de temps ne sont pas [pour eux] des unités de mesure, mais les unités d'un rythme où l'alternance des diversités ramène périodiquement au semblable. En un mot, le calendrier est le code des qualités $d u$ temps. 37

Comment ne pas imaginer, en déportant cette remarque vers un objet aussi temporalisé que l'objet filmique - qui métaphoriquement se présente lui aussi comme un calendrier doté de ses propres qualités -, qu'il ne puisse s'épanouir différemment dans des milieux où se définit une écologie temporelle différente? La question est ouverte.

\section{N O TES}

1. J. Cocteau, Entretiens sur le cinématographe, Paris, Éd. Pierre Belfond, 1973, p. 12-13.

2. G. Genette, La Relation esthétique, Paris, Éd. du Seuil, 1997, p.4-5.

3. J.-C. Passeron, Le Raisonnement sociologique, Paris, Nathan, 1991,

p. 302 .

4. P. Veyne, Le Pain et le Cirque, Paris, Éd. du Seuil, 1976, p. 669.

5. T. Pavel, «L'objet parmi les choses», Critique, n $589-590$, juin-juillet 1996.

6. Que nous avons mis en évidence sur le schéma en opposant termes à termes - en négatif et en positif - les étapes qui mènent de l'histoire racontée à l'histoire reconstituée.

7. N. Élias, Du Temps, Paris, Fayard, 1996 [○ 1984: Über die Zeit], p. 81

8. J.-C. Passeron, Le Raisonnement sociologique, p. 285.

9. On pourrait refaire l'histoire avec des si, il n'empêche que le langage cinématographique des origines, c'est-à-dire du cinéma muet, avait investi une recherche rompue par l'avènement du sonore: il aurait été toutefois sémiologiquement intéressant d'observer comment se serait rationalisé l'équilibre entre images et texte écrit, ce dernier étant une sorte de paramètre incontournable que l'on a congédié sans savoir réellement pourquoi, avec un empressement sans doute trop hâtif. 10. E. Husserl, Leçons pour une phénoménologie de la conscience intime du temps, Paris, P.U.F., 1964

11. J.-C. Passeron et E. Pedler, Le temps donné aux tableaux, documents CERCOM/IMEREC, décembre 1991, p. 11.

12. J.C. Passeron, «Le chassé-croisé des œuvres et de la sociologie", Sociologie de l'art, Paris, La Documentation française, 1986, p. 449.

13. J.-L. Fabiani, «Sur quelques progrès récents de la sociologie des œuvres ", Genèses, n¹1, mars 1993, p. 152.

14. C'est ce que propose pour les œuvres littéraires Pierre Bourdieu dans Les Règles de l'art. Genèse et structure du champ littéraire, Paris, Éd. du Seuil, 1992.

15. On pourrait en effet imaginer cette belle analyse que l'on pourrait engager dans une transposition minutieuse des thèses que R. Moulin développe dans Le Marché de la peinture en France (Paris, Minuit, 1967) ou dans L'Artiste, l'institution et le marché (Paris, Flammarion, 1992). 16. H. R. Jauss, Pour une esthétique de la réception, Paris, Gallimard, 1973.

17. Après G. Lukács, L. Goldmann a développé un programme de sociologie de création littéraire où l'approche marxiste reste le principal moteur de recherche et d'analyse. Ainsi Goldmann écrit: «Les grands écrivains représentatifs sont ceux qui expriment, d'une manière plus ou moins cohérente, une vision du monde qui correspond au maximum de conscience possible d'une classe; c'est le cas partout, pour les philosophes, les écrivains, les artistes".

18. W. Iser, L'Acte de lire, Bruxelles, Éd. Mardaga, 1985.

19. J. Leenhardt, «Théorie de la communication et théorie et la réception", Réseaux, nº6, CNET, 1994, p.41-47. 
20. R. Jakobson, Essais de linguistique générale, Paris, Éd. de Minuit, 1968.

21. Jauss réactive ici une nouvelle conception de l'horizon d'attente qui avait été élaborée par l'herméneutique littéraire de Mannheim.

22. H. R. Jauss, Pour une esthétique de la réception, p. 51.

23. D'après H.-G. Gadamer (Vérité et Méthode, les grandes lignes d'une herméneutique philosophique, Paris, Éd. du Seuil, 1976, p. 147) qui précisa ainsi la notion de fusion: «l'horizon du présent est en formation perpétuelle dans la mesure où il faut perpétuellement mettre à

l'épreuve nos préjugés. C'est d'une telle mise à l'épreuve que relève elle aussi la rencontre avec le passé et la compréhension de la tradition

dont nous sommes issus. L'horizon du présent ne peut donc résolument pas se former sans le passé. Il n'y a plus d'horizon du présent qui puisse exister séparément qu'il n'y a d'horizon historique qu'on puisse conquérir ".

24. H. R. Jauss, Pour une herméneutique littéraire, Paris, Éd. Gallimard, 1988.

25. T.W. Adorno, Théorie esthétique, Paris, Klincksieck, 1989, p. 26-27.

26. Cf. Hippias Majeur et Phèdre.

27. H. R. Jauss, Pour une esthétique de la réception, p. 130.

28. Un argument tente généralement de dénoncer cette recréation incessante des normes et des valeurs: l'existence des classiques qui traversent les époques. Deux réponses ont été avancées: celle de
Gadamer qui prétend qu'il faut chercher l'intemporalité des classiques dans la supériorité des œuvres; celle de Jauss pour qui c'est dans la conciliation renouvelée des horizons des œuvres et de leurs interprètes que la durabilité doit être pensée.

29. L. Wittgenstein dans Leçons et conversations sur l'esthétique, la psychologie et la croyance religieuse, trad. fr., Paris, Gallimard, coll. «Les Essais", 1971.

30. Ibid., p. 76 .

31. Introduction aux comptes rendus d'enquête de J.-C. Passeron et E. Pedler, Le temps donné aux tableaux, p.XIII.

32. J.-C. Passeron, «L'usage faible des images», dans Le Raisonnement sociologique, p. 257-258.

33. J.-C. Passeron, Postface à une exposition, «L'œil et ses maîtres", dans Les Jolis Paysans peints, Marseille, IMEREC, 1989, p. 133.

34. M. Riffaterre se sert de ce principe afin d'identifier ce qu'il appelle des effets de style (Essais de stylistique structurale, Paris, Flammarion, 1971).

35. W. Grossin, Pour une science des temps. Introduction à l'écologie temporelle, Toulouse, Octares, 1996, p. 128.

36. W. Grossin, Les Temps de la vie quotidienne, Berlin, Mouton/De Gruyter, 1974.

37. H. Mendras, La Fin des paysans, Paris, Futuribles Sedeis, 1967, p. 82 . 